\title{
Seleninic acids as chalcogen bond donors: a molecular insight of GPx activity
}

\author{
A. Pizzi ${ }^{1}$, A. Tripathi ${ }^{2,3,4}$, A. Daolio¹, Z. Guo ${ }^{3}$, D. R. Turner ${ }^{3,4}$, G. B. Deacon ${ }^{3,4}$, G. Resnati ${ }^{1}$, H. B. Singh ${ }^{2,4}$ \\ ${ }^{1}$ Department of Chemistry, Materials and Chemical Engineering "Giulio Natta”, Politecnico di Milano, Milano 20131, Italy \\ ${ }^{2}$ Department of Chemistry, Indian Institute of Technology Bombay, Mumbai 400076, India \\ ${ }^{3}$ School of Chemistry, Monash University, Clayton, Victoria 3800, Australia \\ ${ }^{4}$ IITB-Monash Research Academy, Powai, Mumbai 400076, India \\ andrea.pizzi@polimi.it
}

Glutathione peroxidase (GPX) [1] is a selenoenzyme containing multiple selenocysteine units in its active site. It catalyses the reduction of harmful peroxides, thus protecting cells from oxidative stress. High concentrations of active peroxides results in an alternative path of the catalytic cycle of GPX, where selenenic acid residues $(\mathrm{R}-\mathrm{SeOH})$ undergo oxidation to the corresponding seleninic $\left(\mathrm{R}-\mathrm{SeO}_{2} \mathrm{H}\right)$ and selenonic acids $\left(\mathrm{R}-\mathrm{SeO}_{3} \mathrm{H}\right)$. In general, synthetic seleninic acids and their sulfurated analogues sulfinic acids $\left(\mathrm{R}-\mathrm{SO}_{2} \mathrm{H}\right)$ have been reported as key component in redox regulation [2], exerting in some cases a surprising anticancer activity [3].

The reactivity of these moieties may be related to the electrophilic behaviour of selenium and, to a lesser extent, of sulphur. The propensity of an electron rich atom to act as an electrophile is related to the presence of regions of positive electrostatic potential ( $\sigma$ holes) on its surface, located on the back-end of the covalent bonds formed by the considered atom. $\sigma$-hole interactions are named from the group of the periodic table to which the element behaving as an electrophile belongs; based on this, interactions given by atoms of group 16 are dubbed as Chalcogen Bonds (ChB) [4].

Here, we report the controlled oxidation of L-selenocystine $\left(\mathrm{C}_{6} \mathrm{H}_{12} \mathrm{~N}_{2} \mathrm{O}_{4} \mathrm{Se}_{2}\right)$ in selenocysteine seleninic acid, which is a simple mimic of GPX activity. This compound was isolated and single crystals suitable for X-ray diffraction allowed to an insight at the atomic level of the electrophilic behaviour of selenium. This crystal structure suggests the possible involvement of $\mathrm{ChB}$ in the redox regulation activity of the seleninic acid moiety. A survey of the Cambridge Structural Database (CSD) and some computational studies on a small library of these class of compounds may confirm the propensity of seleninic (and sulfinic) acids to act as ChB donors.

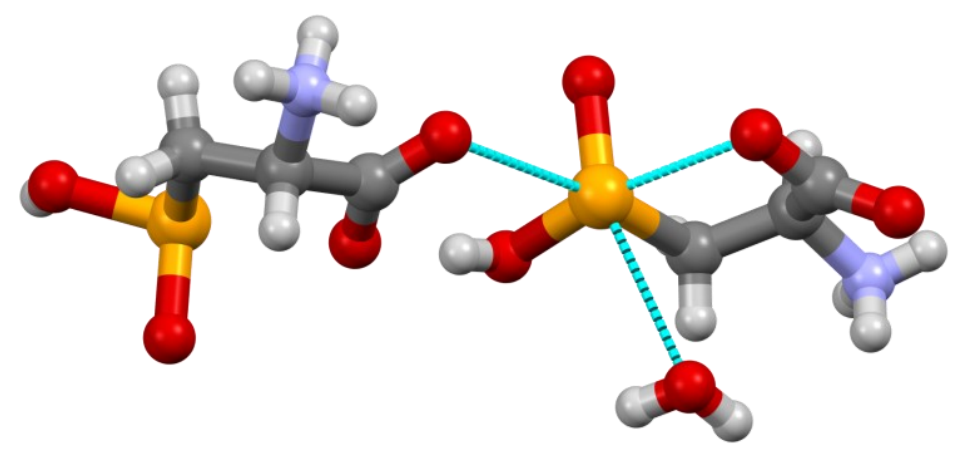

Figure 1. The three ChBs formed by selenocysteine seleninic acid. Color code: carbon, grey; oxygen, red; nitrogen, violet; sulphur, orange; hydrogen, white. ChBs are depicted as light blue dashed lines.

[1] Margis, R., Dunand, C., Teixeira, F. K., Margis-Pinheiro, M. (2008). FEBS J.. 275, 3959.

[2] Jeong, W., Park, S., Chang, T., Lee, D., Rhee, S. (2006). J. Biol. Chem. 281, 14400.

[3] Shen, H., Ding, W., Ong, C.(2002). Free Radic. Biol. Med. 33, 552.

[4] Aakeroy, C. B., Bryce, D. L., Desiraju, G. R., Frontera, A., Legon, A. C., Nicotra, F., Rissanen, K., Scheiner, S., Terraneo, G., Metrangolo, P., Resnati, G. (2019). Pure Appl. Chem., 91, 1889.

Keywords: Selenocistinic acid, Chalcogen Bond, Glutathione peroxidase 